doi: $10.15407 /$ ujpe61.06.0523

A.A. LYOGENKAYA, G.E. GRECHNEV, O.V. KOTLYAR, A.S. PANFILOV, V.P. GNEZDILOV

B. Verkin Institute for Low Temperature Physics and Engineering, Nat. Acad. of Sci. of Ukraine (Kharkiv 61103, Ukraine; e-mail: lyogenkaya@ilt.kharkov.ua)

\title{
ELECTRONIC STRUCTURE
}

PACS $75.85 .+\mathrm{t}, 75.50 . \mathrm{Gg}$, AND MAGNETIC PROPERTIES OF FeTe,

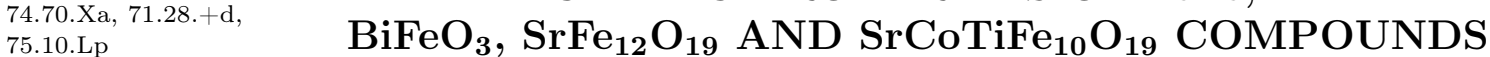

The electronic energy structures and magnetic properties of iron-based compounds with group $V I$ elements ( $\mathrm{FeTe}, \mathrm{BiFeO}_{3}, \mathrm{SrFe}_{12} \mathrm{O}_{19}$ and $\mathrm{SrCoTiFe}_{10} \mathrm{O}_{19}$ ) are studied using the density functional theory (DFT) methods. Manifestations of different types of chemical bonds in magnetism of these compounds are studied theoretically. Calculations of electronic structures of these systems are performed, by using the generalized gradient approximation (GGA) for the description of the exchange and correlation effects within DFT. For $\mathrm{SrFe}_{12} \mathrm{O}_{19}$ and $\mathrm{SrCoTiFe}_{10} \mathrm{O}_{19}$ hexaferrites, the $G G A+U$ method is also employed to deal with strongly correlated $3 d$-electrons. The calculations have revealed distinctive features of the electronic structure of the investigated iron-based compounds with strongly correlated 3d-electrons, which can be responsible for their peculiar structural and magnetic properties.

Ke ywords: electronic structure, magnetic properties, FeTe, $\mathrm{BiFeO}_{3}, \mathrm{SrFe}_{12} \mathrm{O}_{19}$.

\section{Introduction}

The iron-based compounds are usually regarded as strong magnetic materials. However, a new class of iron-based superconductors was recently discovered. The representatives of this class are iron-based compounds with group VI elements, FeS, FeSe, and FeTe, which are distinguished by the simplest crystal structure among iron-based superconductors and by very different magnetic properties [1-6]. The characteristic feature of these compounds is the interplay of antiferromagnetism (AFM) and superconductivity.

Compounds of iron with another element of group VI, oxygen, predominantly belong to ferromagnetic materials. There is a renewed interest in properties of such iron oxides as $\mathrm{BiFeO}_{3}$ - and $\mathrm{MFe}_{12} \mathrm{O}_{19}$-based systems [7-15]. $\mathrm{BiFeO}_{3}$ is antiferromagnetic and mul-

(C) A.A. LYOGENKAYA, G.E. GRECHNEV, O.V. KOTLYAR, A.S. PANFILOV, V.P. GNEZDILOV, 2016

ISSN 2071-0194. Ukr. J. Phys. 2016. Vol. 61, No. 6 tiferroic, whereas $M \mathrm{Fe}_{12} \mathrm{O}_{19}(M=\mathrm{Sr}, \mathrm{Ba}, \mathrm{Pb})$ are ferrimagnetic systems with expected manifestation of multiferroic properties. In these compounds, a substitution of $\mathrm{Fe}$ or the cations with other metals can provide a mixed valence state and very unusual magnetic properties.

The clarification of microscopic mechanisms, which determine electric and magnetic properties of these oxides and chalcogenides, assumes a detailed theoretical study of the electronic structure. A number of electronic structure calculations were carried out for these systems in recent years. However, the data on their electronic energy structure are still incomplete and inconsistent. Moreover, the electronic states of these systems are regarded as strongly correlated, and a proper approach has to be taken for theoretical studies of the electronic structures.

The purpose of this paper is to provide a reliable picture of the electronic band structures and corresponding magnetic properties of $\mathrm{FeTe}, \mathrm{BiFeO}_{3}$, 


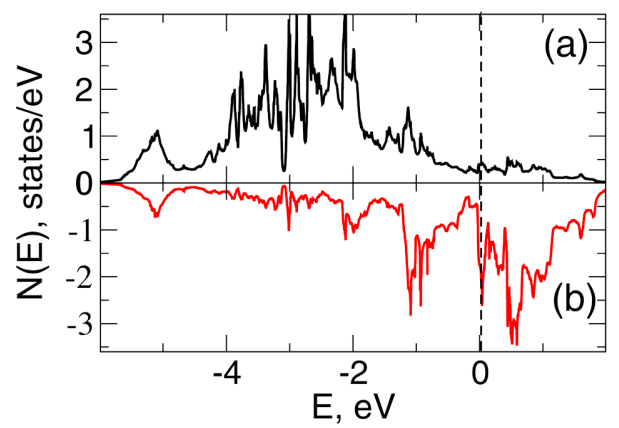

Fig. 1. Spin-polarized densities of states $N(E)$ of FeTe compound for the double-stripe AFM phase (per formula unit): positive $(a)$ and negative $(b)$ values of DOS correspond to majority and minority spin states, respectively. The position of the Fermi level $\left(E_{\mathrm{F}}=0\right)$ is marked by a vertical dashed line

$\mathrm{SrFe}_{12} \mathrm{O}_{19}$, and $\mathrm{SrCoTiFe}_{10} \mathrm{O}_{19}$ compounds. Our stu$\mathrm{dy}$ is based on the density functional theory (DFT) methods, with employing modern extentions to take the effects of correlations into account.

\section{Details of Electronic Structure Calculations}

The self-consistent calculations of electronic structures were carried out, by using the modified relativistic LMTO method with a full potential (FP-LMTO, RSPt implementation [16-18]) and the linearized augmented plane waves method with a full potential (FPLAPW, Elk implementation [19]). The exchange and correlation potentials were treated within the generalized gradient approximation (GGA [20]) of DFT. The spin-orbit coupling was included in the self-consistent calculations.

For the employed full potential FP-LMTO and FPLAPW methods, any restrictions were not imposed on charge densities or potentials of the studied systems, which is especially important for anisotropic layered structures of the investigated compounds. In both methods, the dual basis set was employed to incorporate two valence wave functions with the same orbital quantum number (e.g., $3 p$ and $4 p$ functions for $\mathrm{Fe}$ ). Electronic structure calculations for compounds were carried out for experimental crystal lattice parameters.

\section{Electronic Structure and Magnetic Properties of FeTe}

At temperatures above $70 \mathrm{~K}$, FeTe compound possesses the tetragonal PbO-type crystal structure (space group $P 4 / \mathrm{nmm}$ ). As the temperature decreases, FeTe demonstrates a first-order structural phase transition at $T \simeq 70 \mathrm{~K}$ from the tetragonal to monoclinic structure. This transition is accompanied by the bicollinear AFM ordering, as determined by means of x-ray and neutron diffraction studies [1-4]. In our calculations, we used the experimental data on structural parameters $a, b$ and $c$. The angle between the axes was taken as $90^{\circ}$ (instead of $89.2^{\circ}$ for the actual structure with small monoclinic distortion).

The calculations of the electronic structure are carried out in the GGA-approximation for magnetic phases of FeTe (ferromagnetic, collinear AFM, bicollinear AFM), and a minimum of the total energy is found for the bicollinear or "double stripe" (DS) AFM phase, which appears to be a ground state of FeTe compound. The spin-polarized densities of electronic states (DOS) calculated for the ground state are presented in Fig. 1. As can be seen in Fig. 1, $b$, in the DS AFM phase of FeTe, the Fermi level is located in a very close proximity $(\sim 0.1 \mathrm{eV})$ of the sharp peak of DOS.

The spin-polarized splitting of DOS $N(E)$ (see Fig. 1) provides the formation of magnetic moments at Fe sites for the DS AFM phase of FeTe. The value of magnetic moment $M_{\mathrm{Fe}} \cong 2.37 \mu_{\mathrm{B}}$ obtained in our calculations is in agreement with the results of neutron diffraction studies $\left(M_{\mathrm{Fe}}^{\exp }=2.26 \div 2.54 \mu_{\mathrm{B}}[2,4]\right)$. Such good agreement with experiment confirms the itinerant nature of magnetic moments in FeTe and the adequacy of the DFT-GGA approximation used in the present calculations of magnetic characteristics. The calculated spin density contours in the (001) plane for the AFM phase of FeTe compound are presented in Fig. 2. The double stripe structure of the ground state AFM phase is clearly seen in this figure.

The calculated charge density contours in the vertical (100) plane of a unit cell of FeTe compound are presented in Fig. 3. It demonstrates a substantial anisotropy of the charge density distribution between an iron atom and neighboring atoms of tellurium. It is plausible to assume that the formation of directed covalent bonds between the neighboring $\mathrm{Fe}$ and $\mathrm{Te}$ atoms, seen in Fig. 3, is caused by the hybridization of $d$-states of iron and $p$-states of tellurium.

For a more detailed study of the chemical bonds in FeTe compound, we have calculated the crystal orbital overlap populations (BCOOP, [21]), by using the FP-LMTO method [17]. The calculated $\operatorname{BCOOP}(E)$ 


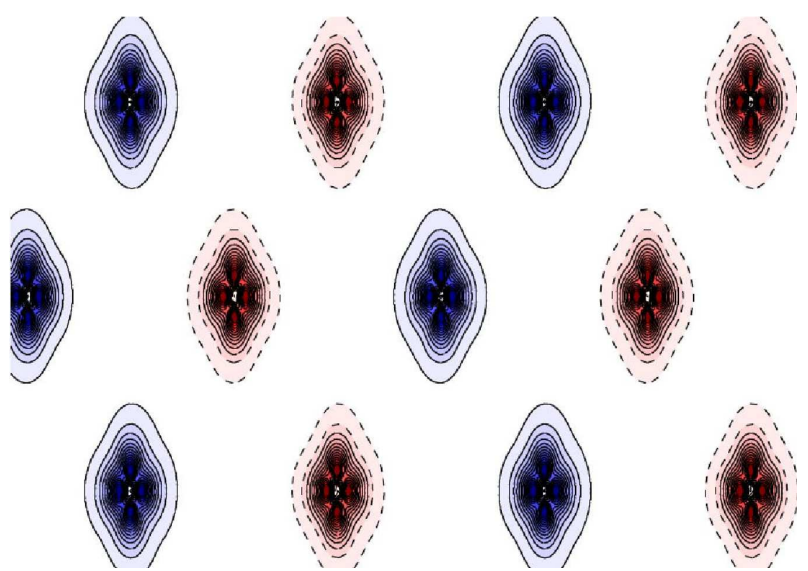

Fig. 2. Calculated spin density contours in the (001) plane for the double-stripe antiferromagnetic phase of FeTe

distributions (see Fig. 4) represent a generalization of the crystal orbital overlap population (COOP) molecular characteristics, known in quantum chemistry, for solids. The values of $\operatorname{BCOOP}(E)$ depend on the energy of electronic states in the valence band and are positive for bonding orbitals and negative for antibonding orbitals, as well as in the case of ionic bonding [21]. The metallic type bonding is characterized by negative values of $\operatorname{BCOOP}(E)$ at energies close to the Fermi level.

According to the results of $\operatorname{BCOOP}(E)$ calculations, the overlap of $\mathrm{Fe}-\mathrm{Te}$ orbitals gives bonding states closer to the bottom of the valence band (positive $\operatorname{BCOOP}(E)$ in the range $-4 \div-2 \mathrm{eV}$, see Fig. $4, b)$. The bonding states are also formed at the $\mathrm{Fe}-\mathrm{Fe}$ orbital overlapping in the energy range $-3 \div-1 \mathrm{eV}$ (Fig. 4, a). In a vicinity of the Fermi energy in the range $-1 \div 1 \mathrm{eV}$ for $\mathrm{Fe}-\mathrm{Te}$ and $\mathrm{Fe}-\mathrm{Fe}$ bonds, the negative values of $\operatorname{BCOOP}(E)$ are obtained (Fig. 4, $a$ and $b$ ). This corresponds to a metallic type of bonding. Basically, the calculated distribution of the electronic density in Fig. 3 definitely has features of metallic bonding, whereas the distinct covalent bonds are formed between $\mathrm{Fe}$ and Te atoms.

In this work, the Fermi surface (FS) of the lowtemperature DS AFM phase of FeTe was calculated for the first time, and it is presented in Fig. 5. The complicated shape of this FS differs drastically from that of paramagnetic FeTe, which was calculated earlier [22] and represents compensating electron and hole cylinders. This radical FS reconstruction at the AFM transition can be the origin of the sign rever-

ISSN 2071-0194. Ukr. J. Phys. 2016. Vol. 61, No. 6

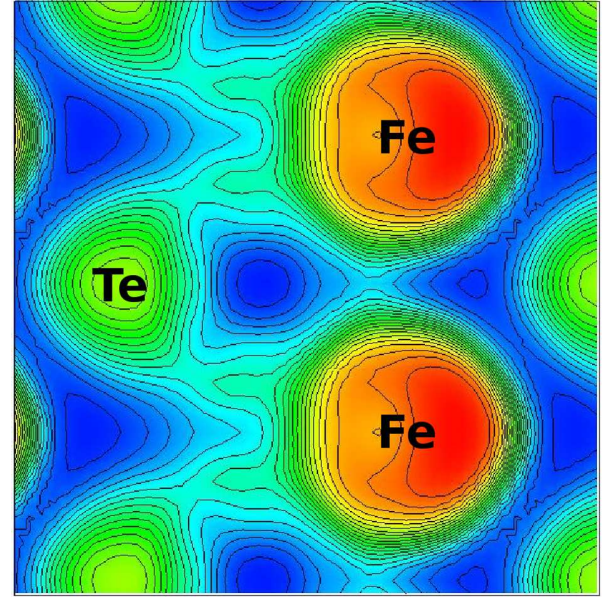

Fig. 3. Calculated charge density contours in the (100) plane for FeTe compound in the DS AFM phase

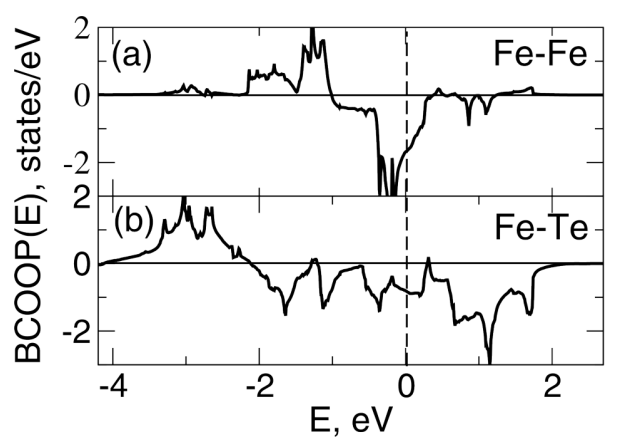

Fig. 4. Balanced crystal orbital overlap populations $\operatorname{BCOOP}(E)$ of FeTe compound in the DS antiferromagnetic phase for the pairs of nearest atoms in the unit cell: $\mathrm{Fe}-\mathrm{Fe}$ $(a)$; Fe-Te $(b)$. The position of the Fermi level $\left(E_{\mathrm{F}}=0\right)$ is indicated by a vertical dashed line

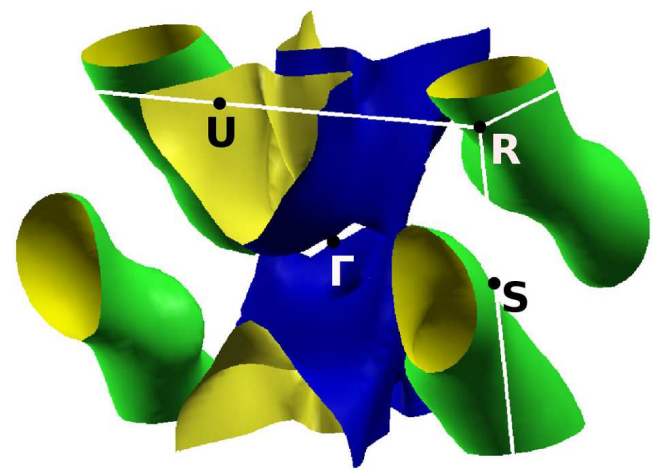

Fig. 5. Calculated Fermi surface of FeTe compound in the bicollinear AFM phase 


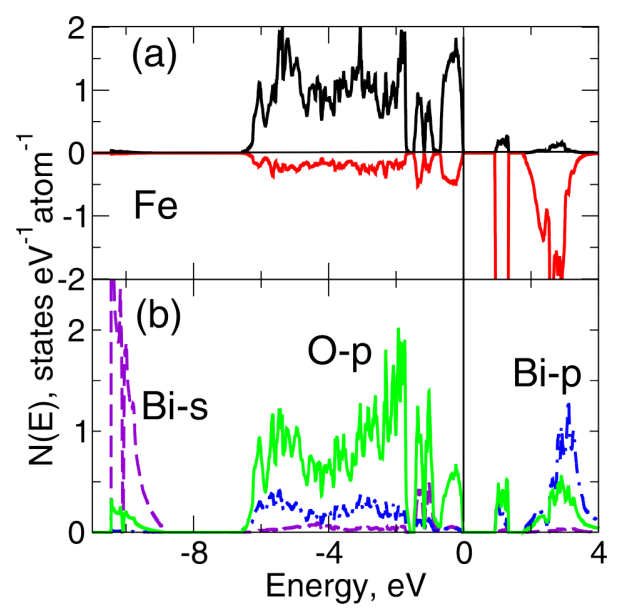

Fig. 6. Partial densities of electronic states $N(E)$ of $\mathrm{BiFeO}_{3}$ in the $G$-type antiferromagnetic phase: spin-polarized densities of states per Fe atom: positive and negative values of DOS correspond to majority and minority spin states, respectively (a); densities of states of $s$ - and $p$-type per Bi atom: dashed and dash-dotted curves, respectively; density of states per oxygen atom: solid curve. The Fermi level $\left(E_{\mathrm{F}}=0\right)$ is indicated by a vertical line $(b)$

sal of the Hall coefficient, which has been observed in FeTe [23]. Due to the multiband electronic structure of FeTe, the AFM transition presumably initiates the reversal of a balance between the electron and hole contributions to the Hall coefficient.

\section{Electronic Structure and Magnetic Properties of $\mathrm{BiFeO}_{3}$}

The compound $\mathrm{BiFeO}_{3}$ is a typical representative of substances known as magnetoelectrics or multiferronics, in which magnetic and electric orderings coexist. Bismuth ferrite $\mathrm{BiFeO}_{3}$ has a relatively simple crystalline structure of the perovskite type and is of interest as a model object for theoretical studies of magnetic and electrical properties. In the last decade, the nonempirical calculations of the electronic structure were performed for different crystalline modifications of bismuth ferrite, and the importance of including the Coulomb correlations in the LSDA $+U$ or GGA $+U$ approximations for a correct description of the electronic structure and ferroelectric properties of $\mathrm{BiFeO}_{3}$ was indicated [24-26]. On the other hand, the possibility of describing the electronic structure and magnetism of $\mathrm{BiFeO}_{3}$ within the density functional theory in the generalized gradient approximation has not been consistently and comprehensively investigated. Here, we have calculated the electronic structure and magnetic properties of $\mathrm{BiFeO}_{3}$ in the DFTGGA approximation, without including the Coulomb parameter $U$, within the FP-LAPW method including the spin-orbital coupling [19].

At low temperatures, $\mathrm{BiFeO}_{3}$ has a rhombohedral distorted perovskite crystal structure (space group $R 3 c$ ). The electronic structure was calculated for the experimental values of crystal lattice parameters, assuming the collinear $G$-type antiferromagnetic ordering [24]. The rhombohedral angle of the $R 3 c$ structure was fixed at its experimental value of $\alpha_{R}=59.35^{\circ}$. The calculated partial electronic densities of states (DOS) $N(E)$ for $\mathrm{BiFeO}_{3}$ in the $G$-type antiferromagnetic phase are shown in Fig. 6. This figure shows that the $G$-type AFM phase is characterized by an insulating state with an energy gap of about $1 \mathrm{eV}$, in good agreement with emission and absorption spectroscopy data [26].

In Fig. 6, b, one can see a narrow band at energies about $-10 \mathrm{eV}$ originating from the $6 s$-states of $\mathrm{Bi}$ and hybridized with the $2 p$-states of oxygen. On the other hand, the $6 p$-states of $\mathrm{Bi}$ are substantially higher in energy. It can be expected that this narrow band, which is predominantly of the $6 s$-states of $\mathrm{Bi}$, corresponds to the so-called stoichiometrically active "lone pair" electrons, which are assumed to be responsible for the polarization in ferroelectrics based on bismuth and lead $[7,8]$. Due to the hybridization with the $2 p$-states of oxygen, this $6 s$ "lone pair" can no longer have a purely spherical spatial charge distribution, but can acquire a component in the form of a "lobe", which is characteristic of $p$-orbitals. Then the $\mathrm{Bi}(s)-\mathrm{O}(p)$ hybridization leads to a noticeable spatial anisotropy in the charge density distribution. Thus, the hybridization of the $s p$-orbitals of $\mathrm{Bi}$ with the $2 p$-orbitals of oxygen leads to the asymmetric charge transfer in the $\mathrm{Bi}-\mathrm{O}$ bonds, which evidently facilitates the development of ferroelectricity.

For a more detailed study of the chemical bonds in $\mathrm{BiFeO}_{3}$, we have calculated the crystal orbital overlap populations (BCOOP, [21]), by using the FPLMTO method [17]. The calculated $\operatorname{BCOOP}(E)$ distributions (see Fig. 7) represent a generalization of the crystal orbital overlap population (COOP) characteristics from quantum chemistry for solids. The values of $\operatorname{BCOOP}(E)$ depend on the energy of electronic states in the valence band and are positive 
for bonding orbitals and negative for antibonding orbitals, as well as in the case of ionic bonding [21].

According to the $\mathrm{BCOOP}(\mathrm{E})$ calculations, the most distinct bonding states in the valence band of $\mathrm{BiFeO}_{3}$ are formed in the $\mathrm{FeO}_{6}$ octahedra upon the hybridization of the $d$-states of iron with the $p$-states of oxygen (Fig. 7, b). This is consistent with the exact coincidence in energy of the dominant regions of the partial densities of the $d$-states of iron (Fig. 6,a) and the $p$ states of oxygen (Fig. 6, b) in the valence band of $\mathrm{BiFeO}_{3}$.

The overlap of the $\mathrm{Bi}-\mathrm{O}$ orbitals yields bonding states near the bottom of the valence band (positive $\operatorname{BCOOP}(E)$ in Fig. 7, $a$ ). It should be noted that the contribution of the $p$-states of $\mathrm{Bi}$ in the valence band is substantially depleted (Fig. 6, $b$ ) due to the charge transfer from $\mathrm{Bi}$ to neighboring $\mathrm{Fe}$ and $\mathrm{O}$ atoms. This can be the origin of the ionic bonding for $\mathrm{Bi}-\mathrm{O}$ orbitals in the upper part of the valence band (see Fig. 7, a).

On the other hand, the ionic bonding is even more pronounced for $\mathrm{Fe}-\mathrm{O}$ bonds near the top of the valence band (Fig. 7, b). The corresponding charge transfer in $\mathrm{Fe}-\mathrm{O}$ pairs provides a distortion of the $\mathrm{FeO}_{6}$ octahedra in $\mathrm{BiFeO}_{3}[7,8]$. In fact, our calculations indicate a predominantly ionic character of the chemical bonds in $\mathrm{BiFeO}_{3}$. The hybridization of $\mathrm{Fe}(d)$ - and $\mathrm{O}(p)$-states also produces a partially covalent component of the $\mathrm{Fe}-\mathrm{O}$ bond.

The antiferromagnetic ordering in $\mathrm{BiFeO}_{3}$ provides the spin-split density of states $N(E)$, and the magnetic moments are predominantly formed at iron ions (see Fig. $6, a$ ). The calculated value of $M_{\mathrm{Fe}} \cong 3.7 \mu_{\mathrm{B}}$ agrees with the data from neutron diffraction studies $\left(M_{\mathrm{Fe}} \cong 3.75 \mu_{\mathrm{B}}[27]\right)$. This good agreement with experiment supports the reliability of the DFT-GGA approach used here, which can be applied to the study of magnetic properties of $\mathrm{BiFeO}_{3}$ under pressure and doping. It is also revealed that a part of the total magnetic moment of the unit cell is distributed among neighboring oxygen ions (up to $0.2 \mu_{\mathrm{B}}$ per $\mathrm{O}$ ion).

\section{Electronic Structure and Magnetic Properties of $\mathrm{SrFe}_{12} \mathrm{O}_{19}$ and $\mathrm{SrCoTiFe}_{10} \mathrm{O}_{19}$}

Hexaferrites $\mathrm{SrFe}_{12} \mathrm{O}_{19}, \mathrm{BaFe}_{12} \mathrm{O}_{19}$, and $\mathrm{PbFe}_{12} \mathrm{O}_{19}$ are known as strong permanent magnets and have been intensively studied for the last decade due to their intriguing magnetic and chemical properties.

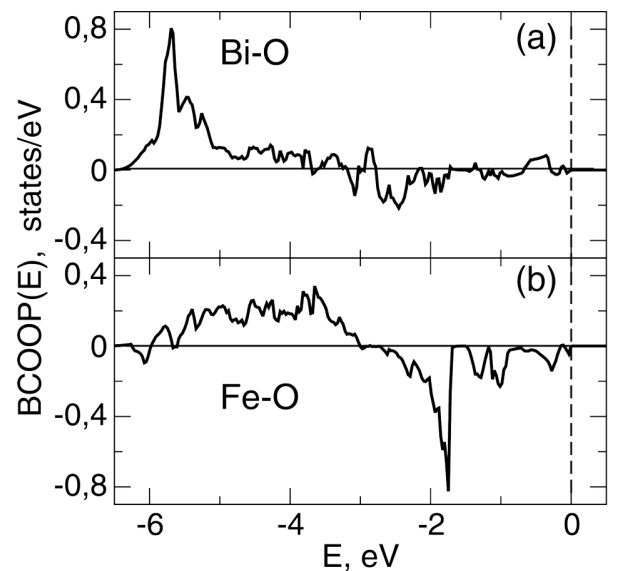

Fig. 7. Crystal orbital overlap populations $\operatorname{BCOOP}(E)$ of $\mathrm{BiFeO}_{3}$ in the G-type antiferromagnetic phase for the pairs of nearest atoms in the unit cell: $\mathrm{Bi}-\mathrm{O}(a), \mathrm{Fe}-\mathrm{O}(b)$. The top of the valence band $(E=0)$ is indicated by a vertical dashed line

Many efforts were undertaken to tune magnetic properties of hexaferrites by substituting $\mathrm{Fe}^{3+}, \mathrm{Ba}^{2+}$ and $\mathrm{Sr}^{2+}$ cations with other transition metal or rare earth ions at various lattice sites (see Refs. [10,13-15] and references therein). Recently, the $\mathrm{SrFe}_{12} \mathrm{O}_{19}$ hexaferrite was found to become magnetoelectric at room temperature by the substitution of $\mathrm{Fe}$ with $\mathrm{Ti}$ and Co $[14,15]$. The aim of the present investigation was, firstly, to find a reliable approach of DFT to describe properly the electronic structure and the magnetism of $\mathrm{SrFe}_{12} \mathrm{O}_{19}$, and then, using this approach, to explore the electronic structure and the magnetic properties of $\mathrm{SrFe}_{12} \mathrm{O}_{19}$-based systems, where $\mathrm{Fe}$ is substituted with $\mathrm{Ti}$ and Co atoms.

Compound $\mathrm{SrFe}_{12} \mathrm{O}_{19}$ possesses the hexagonal crystal structure, which belongs to the $P 6_{3} / m m c$ space symmetry group [10]. The double formula unit cell contains 64 atomic sites distributed among 11 inequivalent Wyckoff positions: $2 d(\mathrm{Sr}), 2 a(\mathrm{Fe}), 2 b$ $(\mathrm{Fe}), 4 f_{1}(\mathrm{Fe}), 4 f_{2}(\mathrm{Fe}), 12 k(\mathrm{Fe}), 4 e(\mathrm{O}), 4 f(\mathrm{O}), 6 h$ $(\mathrm{O}), 12 k_{1}(\mathrm{O})$, and $12 k_{2}(\mathrm{O})$. According to the previous studies $[9,10,12,13]$, the stable spin configuration is assumed to be ferrimagnetic with $\mathrm{Fe}$ at the $4 f_{1}$ and $4 f_{2}$ sites having the magnetic moment antiparallel to the rest of the Fe cations.

It has been established that the LSDA approach has provided incorrectly the metallic ground state of $\mathrm{MFe}_{12} \mathrm{O}_{19}$ hexaferrites [10, 11]. Therefore, for $\mathrm{SrFe}_{12} \mathrm{O}_{19}$, we firstly used the DFT GGA approach [20], which usually gives a more reliable picture of 


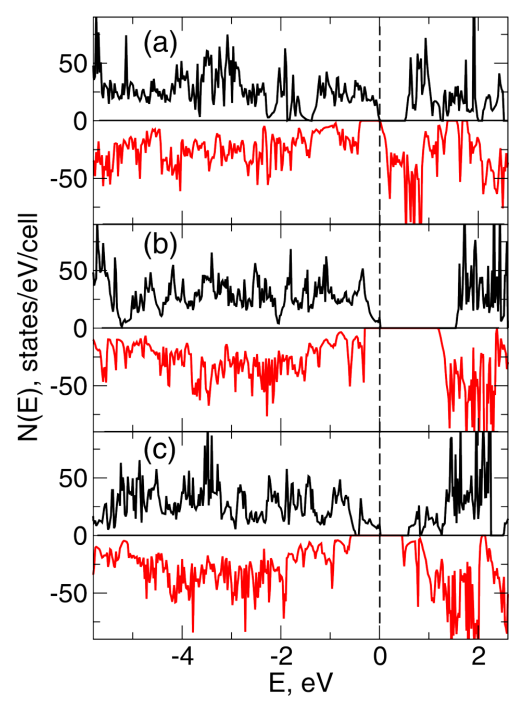

Fig. 8. Total spin-polarized densities of electronic states of strontium hexaferrites: $\mathrm{SrFe}_{12} \mathrm{O}_{19}$, GGA (a); $\mathrm{SrFe}_{12} \mathrm{O}_{19}$, GGA $+U(b) ; \operatorname{SrCoTiFe}_{10} \mathrm{O}_{19}, \mathrm{GGA}+U(c)$. Positive and negative values of DOS correspond to majority and minority spin states, respectively. The Fermi level $\left(E_{\mathrm{F}}=0\right)$ is indicated by a vertical dashed line

Local magnetic moments of ions in different Wyckoff sites of $\mathrm{SrFe}_{12} \mathrm{O}_{19}$ and $\mathrm{SrCoTiFe}_{10} \mathrm{O}_{19}$ calculated, by using GGA and/or GGA $+U$ functionals. Magnetic moments $M$ are given in $\mu_{B}$

\begin{tabular}{|c|c|c|c|c|c|}
\hline \multirow{2}{*}{$\begin{array}{c}\text { Wyckoff } \\
\text { position }\end{array}$} & \multicolumn{3}{|c|}{$\mathrm{SrFe}_{12} \mathrm{O}_{19}$} & \multicolumn{2}{c|}{$\mathrm{SrCoTiFe}_{10} \mathrm{O}_{19}$} \\
\cline { 2 - 6 } & Atom & $M$, GGA & $M$, GGA $+U$ & Atom & $M$, GGA $+U$ \\
\hline \multirow{2}{*}{$2 d$} & $\mathrm{Sr}$ & -0.016 & -0.018 & $\mathrm{Sr}$ & -0.017 \\
$2 a$ & $\mathrm{Fe}$ & 3.748 & 4.052 & $\mathrm{Ti}$ & 0.215 \\
$2 b$ & $\mathrm{Fe}$ & 3.583 & 3.934 & $\mathrm{Co}$ & 2.604 \\
$4 f_{1}$ & $\mathrm{Fe}$ & -3.490 & -3.907 & $\mathrm{Fe}$ & -3.910 \\
$4 f_{2}$ & $\mathrm{Fe}$ & -3.258 & -3.922 & $\mathrm{Fe}$ & -3.911 \\
$12 k$ & $\mathrm{Fe}$ & 3.768 & 4.066 & $\mathrm{Fe}$ & 4.071 \\
$4 e$ & $\mathrm{O}$ & 0.492 & 0.492 & $\mathrm{O}$ & 0.497 \\
$4 f$ & $\mathrm{O}$ & 0.142 & 0.123 & $\mathrm{O}$ & 0.110 \\
$6 h$ & $\mathrm{O}$ & 0.100 & 0.051 & $\mathrm{O}$ & 0.069 \\
$12 k_{1}$ & $\mathrm{O}$ & 0.123 & 0.114 & $\mathrm{O}$ & 0.007 \\
$12 k_{2}$ & $\mathrm{O}$ & 0.217 & 0.218 & $\mathrm{O}$ & 0.220 \\
\hline
\end{tabular}

the ground state for systems with $3 d$-electrons. The GGA $+U$ approach was also employed within the FP-LAPW method [19] in line with Ref. [28] for LSDA $+U$, but with GGA instead of the LSDA exchange-correlation potential. It is believed that this approach provides a better description of the electronic structure for localized electrons in the $3 d$ - transition metal oxides. The on-site Coulomb repulsion energy $U$ was taken close to the previous ab initio estimates [10,13], $U=4 \mathrm{eV}$. The exchange parameter $J$ was assumed to be close to its atomic value $J \simeq 1 \mathrm{eV}[28]$.

The results of GGA and GGA $+U$-based calculations of DOS for $\mathrm{SrFe}_{12} \mathrm{O}_{19}$ compound are presented in Fig. 8, $a$ and $b$, respectively. As can be seen in Fig. 8, a, the GGA FP-LAPW calculations for the spin-polarized ferrimagnetic state have provided the almost zero-gap semiconductor. The energy gaps are enlarged in both majority and minority spin channels, which are moved apart and almost overlaped. For the GGA $+U$ calculations, the insulating ground state was obtained in agreement with experiment (see Refs. [9, 12]) with the energy gap $E_{G} \simeq 1.2 \mathrm{eV}$, as is seen in Fig. 8, b.

The magnetic moments for all inequivalent atoms in the unit cell of $\mathrm{SrFe}_{12} \mathrm{O}_{19}$, which were calculated with both GGA and GGA $+U$ approaches, are listed in Table. For the insulating GGA $+U$ case, the sum of all magnetic moments per unit cell equals $40 \mu_{\mathrm{B}}$, which formally corresponds to $5 \mu_{\mathrm{B}}$ per Fe ion in the adopted ferrimagnetic structure. In fact, however, the theoretical value of $5 \mu_{\mathrm{B}}$ corresponding to the free $\mathrm{Fe}^{3+}$ ion is reduced in a crystal field environment, and a part of the total magnetic moment is transferred to neighboring oxygen ions, as is seen in Table. Actually, the Fe ions in $\mathrm{SrFe}_{12} \mathrm{O}_{19}$ have magnetic moments close to $4 \mu_{\mathrm{B}}$, as it comes from $\mathrm{GGA}+U$ calculations.

The electronic structure of $\mathrm{SrCoTiFe}_{10} \mathrm{O}_{19}$ compound was calculated, by using the GGA $+U$ method. Following the results of previous studies for $3 d-$ transition metal oxides [9, 15], we used $U=4 \mathrm{eV}$ for Fe and $U=3 \mathrm{eV}$ for $\mathrm{Co}$ as the appropriate values for our GGA $+U$ calculations. Based on total energy calculations, we conclude that, in the ground-state configuration, $\mathrm{Ti}$ and Co ions preferentially occupy $2 a$ and $2 b$ positions, respectively. It should be noted that the configuration with $\mathrm{Ti}$ and Co ions in $12 k$ and $4 f_{1}$ positions, respectively, corresponds to a slightly higher total energy and also requires the consideration for the recently synthesized $\mathrm{SrCo}_{2} \mathrm{Ti}_{2} \mathrm{Fe}_{8} \mathrm{O}_{19}$ multiferroic compound [15].

Our GGA $+U$ calculations have revealed the insulating ground state for $\mathrm{SrCoTiFe}_{10} \mathrm{O}_{19}$ with the energy gap $E_{G} \simeq 0.5 \mathrm{eV}$, as is seen in Fig. 8, c. The magnetic moments on Co atoms are found to be $2.6 \mu_{\mathrm{B}}$ 
(see Table), which is lower than that corresponding to a high-spin state of $\mathrm{Co}^{2+}$ ion. As in the case of $\mathrm{Fe}^{3+}$ ions, the high value of moment corresponding to a free $\mathrm{Co}^{2+}$ ion is reduced in the crystal field, and the total magnetic moment is partly distributed among neighboring oxygen ions, as is seen in Table. The sum of all magnetic moments per unit cell of $\mathrm{SrCoTiFe}_{10} \mathrm{O}_{19}$ equals $28 \mu_{\mathrm{B}}$.

\section{Conclusions}

The results of the present GGA DFT calculations indicate the predominantly metallic character of the chemical bonding in FeTe compound, with partially covalent components of $\mathrm{Fe}-\mathrm{Te}, \mathrm{Te}-\mathrm{Te}$ and $\mathrm{Fe}-\mathrm{Fe}$ bonds. In fact, the hybridization of the $d$-states of iron with the $p$-states of tellurium results in a pronounced anisotropy of the spatial distribution of charge density in the area between the planes of $\mathrm{Fe}$ and $\mathrm{Te}$, as well as in the $\mathrm{Te} \rightarrow \mathrm{Fe}$ charge transfer. The presence of the covalent bonding presumably promotes the stabilization of monoclinic structural distortions of the tetragonal phase of FeTe at low temperatures.

The results of calculations affirm that the magnetic properties of FeTe are well described within the approach of itinerant electrons and GGA DFT. In particular, it is established that the bicollinear AFM phase has the lowest total energy, being the ground state of FeTe compound. The calculated value of magnetic moment for the bicollinear AFM phase $\left(M_{\mathrm{Fe}} \cong\right.$ $\left.\cong 2.4 \mu_{\mathrm{B}}\right)$ is very close to the result of neutron diffraction experiments.

On the other hand, in $\mathrm{BiFeO}_{3}$ compound, the GGA DFT calculations have revealed that the chemical bonding is predominantly of an ionic character with partial covalent components of the $\mathrm{Bi}-\mathrm{O}$ and $\mathrm{Fe}-$ $\mathrm{O}$ bonds. For the purely ionic bonding, the spatial distribution of the charge would be purely spherical near the $\mathrm{Bi}, \mathrm{Fe}$, and $\mathrm{O}$ ions with the keeping of an ideal perovskite structure. A covalent bonding facilitates the stabilization of structural distortions, which favors the formation of a ferroelectric phase in $\mathrm{BiFeO}_{3}$. In particular, the hybridization of the $s, p$ states of $\mathrm{Bi}$ with the $2 p$-states of oxygen leads to a distinct spatial anisotropy of the charge density distribution and to an asymmetry in the charge transfer in $\mathrm{Bi}-\mathrm{O}$ bonds, which presumably causes the ferroelectric polarization in $\mathrm{BiFeO}_{3}$. On the other hand, the hybridization of $\mathrm{Fe}(d)$ - and $\mathrm{O}(p)$-states leads to a substantial spin polarization in the AFM $G$-phase and to a distortion and a turn of the $\mathrm{FeO}_{6}$ octahedra. This hybridization reduces the value of magnetic moment of free $\mathrm{Fe}^{3+}$ ion to $M_{\mathrm{Fe}} \cong 3.7 \mu_{\mathrm{B}}$, and it is also responsible for the spin polarization of electronic states of oxygen ions, providing the noticeable magnetic moment $M_{O} \simeq 0.1 \mu_{\mathrm{B}}$ for each $\mathrm{O}$ ion.

The electronic structures of hexagonal strontium ferrites $\mathrm{SrFe}_{12} \mathrm{O}_{19}$ and $\mathrm{SrCoTiFe}_{10} \mathrm{O}_{19}$ are also calculated in the generalized gradient approximation of DFT. However, the GGA $+U$ method has been employed to improve the description of localized $3 d$ electrons and to reproduce the insulating ground states of these compounds, with the energy gaps about $1 \mathrm{eV}$. Resultantly, the calculated magnetic moments of $3 d$-atoms are notably lower than the highspin values of moments of free $\mathrm{Fe}^{3+}$ and $\mathrm{Co}^{2+}$ ions. In fact, these moments are reduced in the crystal field environment, and the total magnetic moment is partly distributed among neighboring oxygen ions. The total magnetic moment of ferrimagnetic $\mathrm{SrCoTiFe}_{10} \mathrm{O}_{19}$ depends on the occupancy of particular sites with the substitution of $\mathrm{Fe}^{3+}$ ions by $\mathrm{Co}^{2+}$ and $\mathrm{Ti}^{4+}$.

The results of the present work indicate that the theoretical analysis of electronic structures of ironbased compounds with group VI elements (chalcogenides and oxygen) by means of GGA and GGA $+U$ DFT calculations provides a way to explore peculiar magnetic properties of different systems with strongly correlated electrons. This includes superconductors with AFM ordering, various multiferroics, and strong ferrimagnets.

This work was supported by the Russian-Ukrainian RFBR-NASU project 78-02-14 and was performed with the use of the computational facilities of a gridcluster ILTPE at B. Verkin Institute for Low Temperature Physics and Engineering of the National Academy of Sciences of Ukraine.

1. Y. Mizuguchi and Y. Takano, J. Phys. Soc. Jpn. 79, 102001 (2010); doi:10.1143/JPSJ.79.102001.

2. A. Martinelli, A. Palenzona, M. Tropeano, C. Ferdeghini, M. Putti, M.R. Cimberle, T.D. Nguyen, M. Affronte, and C. Ritter, Phys. Rev. B 81, 094115 (2010); doi:10.1103/PhysRevB.81.094115.

3. R. Viennois, E. Giannini, D. van der Marel, and R. Črný, J. Solid State Chem. 183, 769 (2010); doi:10.1016/j.jssc.2010.01.024. 
4. S. Li, C. de la Cruz, Q. Huang, Y. Chen, J.W. Lynn, J. Hu, Y.-L. Huang, F.-C. Hsu, K.-W. Yeh, M.-K. Wu, and P. Dai, Phys. Rev. B 79, 054503 (2009); doi:10.1103/PhysRevB.79.054503.

5. G.E. Grechnev, A.S. Panfilov, A.V. Fedorchenko, V.A. Desnenko, S.L. Gnatchenko, V. Tsurkan, J. Deisenhofer, A. Loidl, D.A. Chareev, O.S. Volkova, and A.N. Vasiliev, J. Magn. Magn. Mater. 324, 3460 (2012); doi:10.1016/j.jmmm.2012.02.065.

6. X. Chen, P. Dai, D. Feng, T. Xiang, and F.-C. Zhang, Nat. Sci. Rev. 1371 (2014); doi:10.1093/nsr/nwu007.

7. G. Catalan and J.F. Scott, Adv. Mater. 21, 2463 (2009); doi:10.1002/adma.200802849.

8. K.F. Wang, J.-M. Liu, and Z.F. Ren, Adv. Phys. 58, 321 (2009); doi:10.1080/00018730902920554.

9. G.F. Dionne, Magnetic Oxides (Springer, Berlin, 2009); doi:10.1007/978-1-4419-0054-8.

10. P. Novak, K. Knizek, M. Kupferling, R. Grossinger, and M.W. Pieper, Eur. Phys. J. B 43, 509 (2005); doi:10.1140/epjb/e2005-00084-8.

11. P. Novak and J. Rusz, Phys. Rev. B 71, 184433 (2005); doi:10.1103/PhysRevB.71.184433.

12. R.C. Pullar, Prog. Mater. Sci. 57, 1191 (2012); doi:10.1016/j.pmatsci.2012.04.001.

13. L.S.I. Liyanage, S. Kim, Y.-K. Hong, J.-H. Park, S.C. Erwin, and S.-G. Kim, J. Magn. Magn. Mater. 348, 75 (2013); doi:10.1016/j.jmmm.2013.08.006.

14. K. Ebnabbasi, M. Mohebbi, and C. Vittoria, J. Appl. Phys. 113 17C703 (2013); doi:10.1063/1.4793606.

15. M. Feng, B. Shao, Y. Lu, and X. Zuo, J. Appl. Phys. 115 17D908 (2014); doi:10.1063/1.4865886.

16. G.E. Grechnev, Low Temp. Phys. 35, 638 (2009); doi:10.1063/1.3224723.

17. J.M. Wills, M. Alouani, P. Andersson, A. Delin, O. Eriksson, and A. Grechnev, Full-Potential Electronic Structure Method. Energy and Force Calculations with Density Functional and Dynamical Mean Field Theory (Springer, Berlin, 2010); doi:10.1007/978-3-642-15144-6.

18. http://fplmto-rspt.org/.

19. http://elk.sourceforge.net/.

20. J.P. Perdew, K. Burke, and M. Ernzerhof, Phys. Rev. Lett. 77, 3865 (1996); doi:10.1103/PhysRevLett.77.3865.

21. A. Grechnev, R. Ahuja, and O. Eriksson, J. Phys. Condens. Matter 15, 7751 (2003); doi:10.1088/0953$8984 / 16 / 29 / 015$.
22. A. Subedi, L. Zhang, D.J. Singh, and M.H. Du, Phys. Rev. B 78, 134514 (2008); doi:10.1103/PhysRevB.78.134514.

23. Y. Liu, R.K. Kremer, and C.T. Lin, Supercond. Sci. Technol. 24, 035012 (2011); doi:10.1088/09532048/24/3/035012.

24. J.B. Neaton, C. Ederer, U.V. Waghmare, N.A. Spaldin, and K.M. Rabe1, Phys. Rev. B 71, 014113 (2005); doi:10.1103/PhysRevB.71.014113.

25. S.J. Clark and J. Robertson, Appl. Phys. Lett. 90132903 (2007); doi:10.1063/1.2716868.

26. J.A. McLeod, Z.V. Pchelkina, L.D. Finkelstein, E.Z. Kurmaev, R.G. Wilks, A. Moewes, I.V. Solovyev, A.A. Belik, and E. Takayama-Muromachi, Phys. Rev. B 81, 144103 (2010); doi:10.1103/PhysRevB.81.144103.

27. I. Sosnowska, W. Schafer, W. Kockelmann, K.H. Andersen, and I.O. Troyanchuk, Appl. Phys. A 74, [Suppl.], S1040 (2002); doi:10.1007/s003390201604.

28. A.I. Liechtenstein, V.I. Anisimov, and J. Zaanen, Phys. Rev. B 52, 5467 (1995); doi:10.1103/PhysRevB.52.R5467.

Received 23.09.15

А.А. Легенъка, Г.С. Гречнєв,

О.В. Котляр, А.С. Панфілов, В.П. Гнезділов

ЕЛЕКТРОННА СТРУКТУРА

ТА МАГНІТНІ ВЛАСТИВОСТІ СПОЛУК FеTе,

$\mathrm{BiFeO}_{3}, \mathrm{SrFe}_{12} \mathrm{O}_{19} \mathrm{I} \mathrm{SrCoTiFe}_{10} \mathrm{O}_{19}$

$\mathrm{P}$ е з ю м е

Електронні енергетичні структури та магнітні властивості сполук заліза з елементами VI групи (FeTe, $\mathrm{BiFeO}_{3}$, $\mathrm{SrFe}_{12} \mathrm{O}_{19}$ та $\mathrm{SrCoTiFe}_{10} \mathrm{O}_{19}$ ) були досліджені з використанням методів теорії функціонала густини (DFT). Теоретично вивчені прояви різних типів хімічного зв'язку в магнетизмі цих сполук. Розрахунки електронних структур цих систем були проведені з використанням наближення узагальненого градієнта (GGA) для описання обмінно-кореляційних ефектів в рамках DFT. Для гексаферитів $\mathrm{SrFe}_{12} \mathrm{O}_{19}$ i $\mathrm{SrCoTiFe}_{10} \mathrm{O}_{19}$ також використовувався метод GGA $+U$ з метою урахування сильних кореляцій $3 d$-електронів. Розрахунки дозволили виявити особливості електронної структури сполук на основі заліза з сильно корельованими $3 d-$ електронами, які відповідають за структурні та магнітні властивості цих систем. 